\title{
A Rare Case of Pulmonary Epithelioid Hemangioendothelioma Presenting with Skin Metastasis
}

\author{
Hyung-Suk Ro ${ }^{1}$, Jin Yong Shin ${ }^{1}$, Si-Gyun Roh ${ }^{1}$, Nae-Ho Lee ${ }^{1}$, Kyung-Moo Yang ${ }^{1}$, \\ Woo-Sung Moon ${ }^{2}$ \\ Departments of ${ }^{1}$ Plastic and Reconstructive Surgery and ${ }^{2}$ Pathology, Chonbuk National University Medical School, Jeonju, Korea
}

\begin{abstract}
Epithelioid hemangioendothelioma (EHE) is a well-differentiated and rare vascular tumor. Systemic metastases are uncommon. Herein, we present a patient with skin metastasis of pulmonary EHE (PEH) that was treated by wide excision. A 76-year-old male was evaluated due to pulmonary thromboembolism and a solitary pulmonary nodule. A biopsy was performed and pathological examination of the mass confirmed EHE. No metastasis was observed. The patient returned to care approximately two years later due to a painful nodule in the right lower leg. A skin biopsy showed metastatic EHE from the lung. We used a safety margin of $1 \mathrm{~cm}$ based on clinical experience, because no prior case had been reported regarding the resection margin appropriate for primary cutaneous EHE and skin metastases of PEH. At four months after surgery, the patient recovered without complications or recurrence. Skin metastasis of PEH is extremely rare, and only two cases have been reported in the literature. In this case, we report a rare case of PEH with histologically diagnosed skin metastasis that was successfully treated by curative resection. It is expected that this case report will provide a helpful contribution to the extant data regarding PEH metastases.
\end{abstract}

Keywords Hemangioendothelioma, epithelioid / Skin / Cutaneous surgery
Correspondence: Jin Yong Shin Department of Plastic and Reconstructive Surgery, Chonbuk National University Medical School, 20 Geonji-ro, Deokjin-gu, Jeonju 54907, Korea

Tel: +82-63-250-1860

Fax: +82-63-250-1866

E-mail:psjyshin@gmail.com
No potential conflict of interest relevant to this article was reported.

\section{INTRODUCTION}

Epithelioid hemangioendothelioma (EHE) is a well-differentiated, rare vascular tumor, with a wide spectrum of behavior and an epithelioid and histiocytoid appearance. It originates from pre-endothelial or vascular endothelial cells, represents less than $1 \%$ of all vascular neoplasms, and was described for the first time in 1975 by Dail et al. as pulmonary epithelioid hemangioendothelioma (PEH) [1]. It is well known that the disease is fairly rare; the estimated prevalence of EHE is less than one in
$1,000,000$, with only approximately 248 cases of $\mathrm{PEH}$ reported in the current literature [2]. Involvement of the liver can occur, but systemic metastases are uncommon [3].

Herein, we present a patient with skin metastasis of $\mathrm{PEH}$ that was treated by wide excision and coverage by split-thickness skin grafting.

\section{CASE}

A 76-year-old male was referred to our hospital by a local clinic, 
with a preliminary diagnosis of pulmonary thromboembolism and a solitary pulmonary nodule. In the chest computed tomography (CT) performed at our hospital, an approximately 9-mm lobar nodule was observed (Fig. 1). A biopsy was performed and the pathological examination of the mass confirmed EHE. Immunohistochemistry was positive for $\mathrm{CD} 31$, focally positive ( $>5 \%$ ) for ki67, and negative for CK. Metastasis was not discovered. A right lower lobectomy and lymph node dissection were then performed. The patient revisited our dermatology department due to a painful pruritic localized erythematous umbilicated nodule in the right lower leg approximately two years after his discharge (Fig. 2). The skin lesion was suspected to be a hypertrophic scar or granuloma. A skin biopsy was positive for CD31, CD34, factor VIII-related antigen, and S100 protein, confirming metastatic EHE from the lung. Subsequent evaluation of metastasis including fludeoxyglucose positron emission tomography/CT, chest $\mathrm{CT}$, and brain magnetic resonance imaging did not discover any foci of metastasis. The patient was referred to the plastic surgery department, and was scheduled for elective surgical resection. Under spinal anesthesia, the patient underwent a wide complete excision with splitthickness skin grafting. Intraoperative examination of the mass in the right lower leg indicated that it was approximately $3 \times 3.5$ $\mathrm{cm}$ in size. We used a safety margin of $1 \mathrm{~cm}$ based on clinical experience, because no prior case had been reported regarding the appropriate resection margin for primary cutaneous EHE and

Fig. 1. Chest computed tomography at the time of hospital admission

A 9-mm intermediate lobular nodule was seen (red arrow) in the subpleura of the right lower lung.

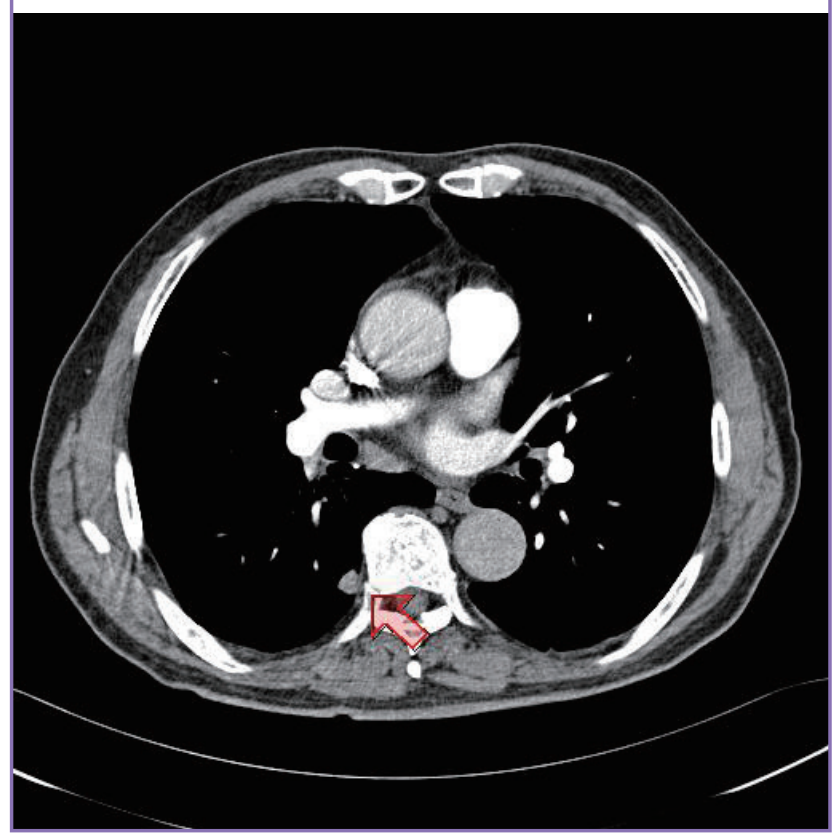

skin metastases of $\mathrm{PEH}$.

The resected specimen was approximately $3.9 \times 3.8 \times 1.1 \mathrm{~cm}$, and the gross appearance of the skin exhibited a whitish elevated lesion that measured $1.6 \times 1.4 \mathrm{~cm}$ (Fig. 3). Histopathologic images showed that the center of the tumor was often fibrotic and parvicellular, but the periphery of tumor had higher cellularity. The boundaries were grossly defined because peripheral cellularity was increased. Moreover, endothelial cells were distributed widely over the normal tissue, and the boundaries were therefore not clearly defined histopathologically. The tumor cells consisted of nests of epithelioid cells, having a somewhat glassy eosinophilic cytoplasm and relatively uniform nuclei. These cells were similar to the previous lung lesion, with epithelioid-like features, and did not show prominent nuclear atypia or mitosis. The cells were positive for the endothelial marker CD34 (Fig. 4). At six months after surgery, no deformity remained, and the

Fig. 2. Preoperative photograph showing the tumor mass

An approximately $3 \times 3-\mathrm{cm}$ localized erythematous umbilicated nodule was found on the right lower leg.

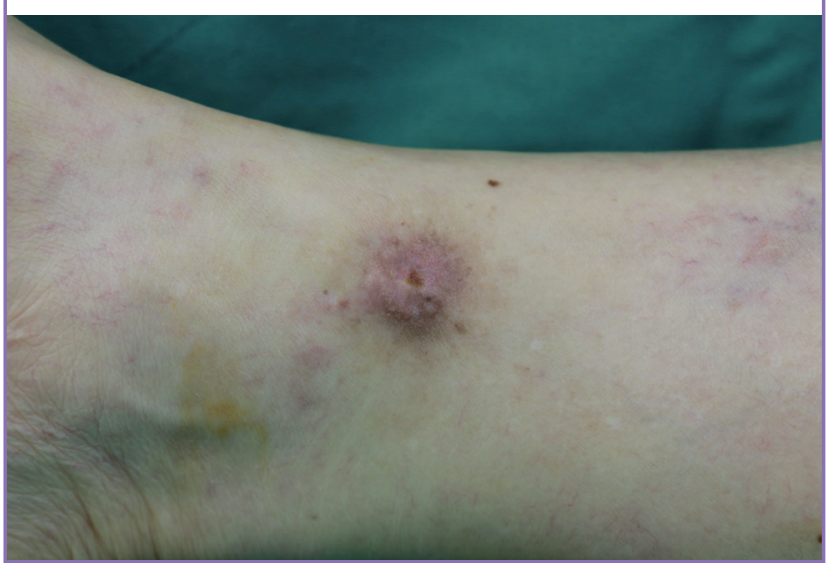

Fig. 3. Intraoperative photograph showing the tumor mass

The resected specimen measured approximately $3.9 \times 3.8 \times 1.1 \mathrm{~cm}$ and the gross appearance of the skin exhibited a whitish elevated lesion that measured $1.6 \times 1.4 \mathrm{~cm}$

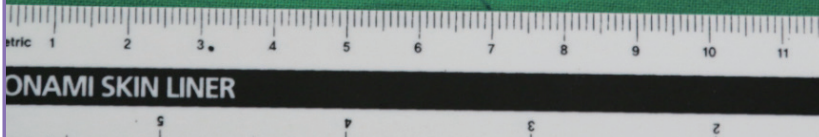




\section{Fig. 4. Microscopic findings of the mass}

(A) The center of tumor was often fibrotic and parvicellular, but the periphery of the tumor had higher cellularity $\left(H \& E_{1} \times 40\right)$. (B) The tumor cells consisted of nests of epithelioid cells, with somewhat glassy eosinophilic cytoplasm and relatively uniform nuclei. Nuclear atypia and mitosis were not prominent $\left(H \& E_{1} \times 200\right)$. (C) Immunohistochemical stains were positive for CD34 $(\times 100)$.

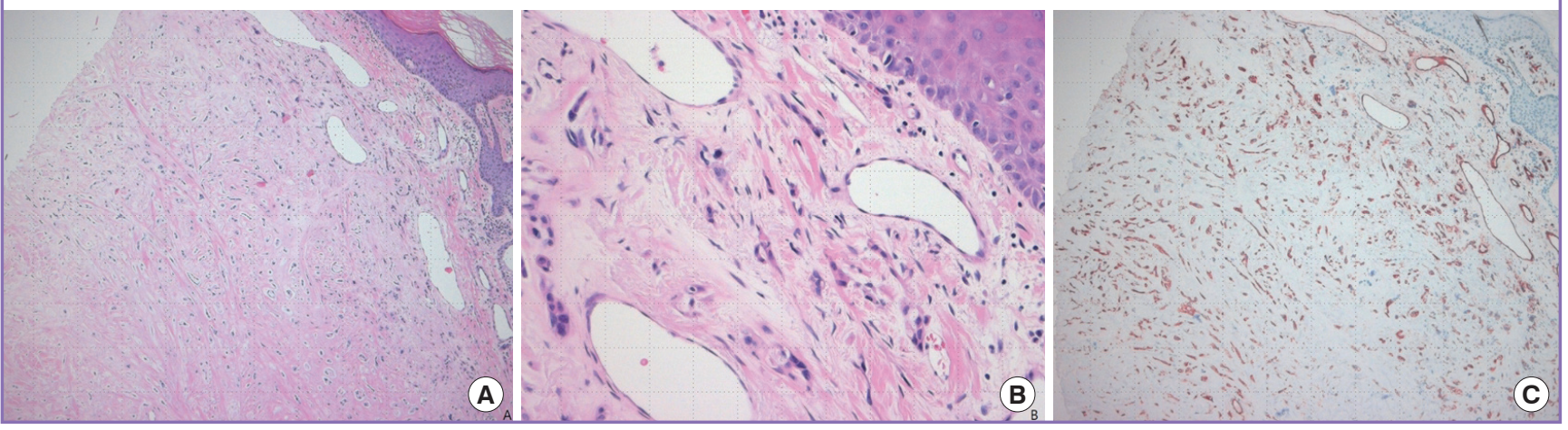

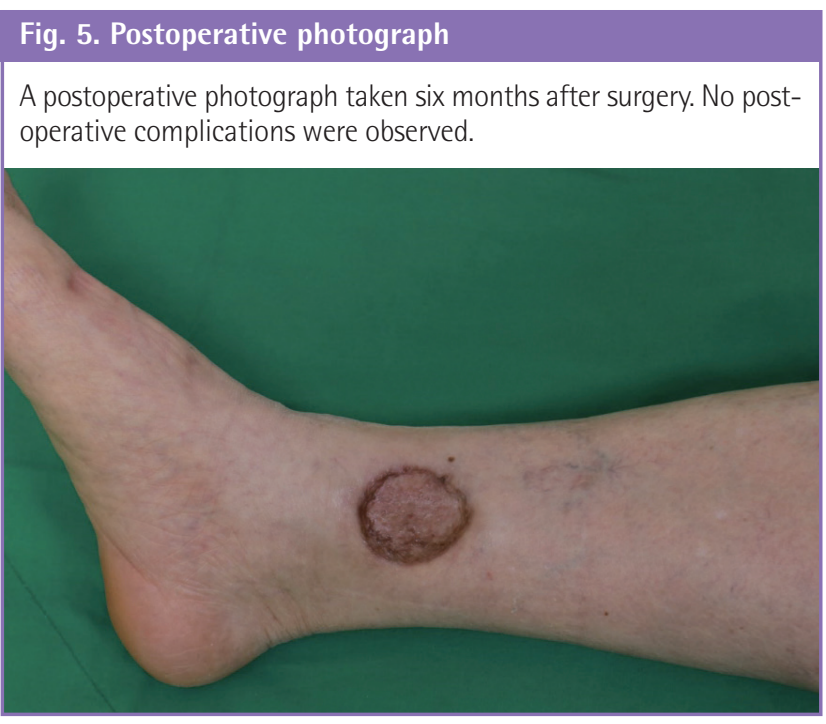

patient recovered without complications or recurrence (Fig. 5).

\section{DISCUSSION}

Vascular neoplasms with borderline characteristics between benign hemangiomas and highly malignant angiosarcomas are called hemangioendotheliomas. The following subtypes of this neoplasm have been identified: papillary intralymphatic angioendothelioma (or Dabska tumor), retiform hemangioendothelioma, kaposiform hemangioendothelioma, epithelioid hemangioendothelioma, pseudomyogenic hemangioendothelioma (or epithelioid sarcoma-like hemangioendothelioma), and composite hemangioendothelioma [4]. In 1982, Weiss and Enzinger first used the term epithelioid hemangioendothelioma to describe a vascular bone and soft tissue tumor with intermediate malignancy features between angiosarcoma and hemangioma and an important endothelial or histocytic component as a sub- type of hemangioendothelioma [1]. Subsequently, EHE has been considered to be a low-grade malignant vascular neoplasm with low metastatic potential but a high tendency to recur, although in a recent series of 30 cases of cutaneous EHE, the follow-up of 24 cases showed local recurrence in three cases and systemic metastasis in five cases, with four patients dying of the tumor, suggesting that EHE of the skin and soft tissues should be regarded as a fully malignant tumor rather than a borderline vascular neoplasm, although with a better prognosis than conventional angiosarcoma. In the recently revised World Health Organization classification of soft-tissue tumors, it has been placed under the heading of intermediate endothelial tumors [5]. Based on the above considerations, it could be difficult to identify the primary lesion when metastases to other organs are observed, as EHE may come from either tissue and metastasize to the other or originate from more than one primary organ. Despite its apparent multicentric origin, it is unusual for it to expand outside of the pulmonary system [3]. PEH dissemination may occur within the pleural cavity, lymphatic vessels, and blood vessels. Distant hematogenous metastases have been found mostly in the liver, but also in the lymph nodes, brain, peritoneum, and bone. However, skin metastasis of PEH is extremely rare, and only two cases have been reported in the current literature [2]. Unlike previous reports, the patient in our case was diagnosed with PEH, and was successfully treated with lobectomy and recovered without complications or evidence of metastasis. Our case is especially significant due to the successful treatment of the ensuing skin lesion, which was histologically diagnosed as a skin metastasis, through complete surgical excision.

Ordinarily, when the lung and other organs are involved simultaneously, it may be hard to decide which is the primary tumor. However, in our case, an extrapulmonary lesion was found after the resection of the primary lung mass and the histologic diag- 
nosis was confirmed. Thus, we concluded that the extrapulmonary lesion reflected metastasis of the PEH.

In this report, we present a rare case of PEH with histologically diagnosed skin metastasis that was successfully treated by curative surgical resection. We expect that this case will provide data relevant for understanding the metastatic behavior of $\mathrm{PEH}$.

\section{REFERENCES}

1. Sardaro A, Bardoscia L, Petruzzelli MF, et al. Pulmonary epithelioid hemangioendothelioma presenting with vertebral metastases: a case report. J Med Case Rep 2014;8:201.
2. Sardaro A, Bardoscia L, Petruzzelli MF, et al. Epithelioid hemangioendothelioma: an overview and update on a rare vascular tumor. Oncol Rev 2014;8:259.

3. Yanagawa $\mathrm{H}$, Hashimoto $\mathrm{Y}, \mathrm{Bando} \mathrm{H}$, et al. Intravascular bronchioloalveolar tumor with skin metastases. Chest 1994;105: 1882-4.

4. Requena L, Kutzner H. Hemangioendothelioma. Semin Diagn Pathol 2013;30:29-44.

5. Carranza-Romero C, Molina-Ruiz AM, Perna Monroy C, et al. Cutaneous epithelioid hemangioendothelioma on the sole of a child. Pediatr Dermatol 2015;32:e64-9. 